M. MARINI AND G. DE PHILIPPIS

KODAI MATH. J.

37 (2014), 586-594

\title{
A NOTE ON PETTY'S THEOREM
}

\author{
Michele Marini and Guido de PhilipPis
}

\begin{abstract}
In this short note we show how, by exploiting the regularity theory for solutions to the Monge-Ampère equation, Petty's equation characterizes ellipsoids without assuming any a priori regularity assumption.
\end{abstract}

\section{Introduction}

Affine inequalities play a very important role in the study of the geometry of convex bodies and they also find applications in several different fields (e.g. ordinary and partial differential equation, functional analysis). In [13] Petty treated three closely related affine problems, namely the Blaschke-Santalò inequality, the affine isoperimetric inequality and the geominimal surface area inequality ${ }^{1}$, and he characterized ellipsoids as the only extremal bodies for these inequalities. In order to establish this characterization he proved that if $K \subset \mathbf{R}^{N}$ is an extremal convex body for these inequalities, then necessarily there must exist a positive constant $c_{K}$ such that

$$
f_{K}(\omega)=c_{K} h_{K}^{-N-1}(\omega),
$$

for every $\omega \in \mathbf{S}^{N-1}$. Here

$$
h_{K}(\omega)=\max \{\omega \cdot x: x \in K\}
$$

denotes the support function of the convex body $K$ and $f_{K}$ is the curvature function of $K$, see Section 2.1.

Petty was then able to show that (1.1) implies that $K$ is an ellipsoid if $N=2$. If $N \geq 3$ he obtained the same result only under the assumption that $K$ is a $C^{2}$-regular convex body or that $K$ is a body of revolution. In any case this was sufficient to prove that extremal sets for the above mentioned problems are ellipsoids since symmetrization techniques allow to reduce to the case of axially symmetric sets.

2010 Mathematics Subject Classification. 52A20, 52A40, 53C45, 35B65.

Key words and phrases. Convex bodies, affine inequalities, Monge-Ampère.

${ }^{1}$ For the position of these problems and a comprehensive overview on the subject we refer to [6] and [13] and the references therein.

Received April 4, 2014; revised April 18, 2014. 
It remains however an interesting question to understand to which extent (1.1) characterizes ellipsoids without assuming any a priori regularity assumption on $K$, see for instance [6]. In this short note we prove that every convex body satisfying (1.1) is actually an ellipsoid. More precisely we prove

THEOREM 1.1. Let $K$ be a convex body which possesses a curvature function $f_{K}$; if equation (1.1) is satisfied for some positive constant $c_{K}$, then $K$ is an ellipsoid.

Besides its own interest Theorem 1.1 will have applications in several problems in which (1.1) characterizes extremal bodies, but for which it is a priori unknown their regularity as well as it can provide new short proofs of some results in which (1.1) appears. As examples let us quote $[9,21,7,8]$ concerning, respectively, convolution bodies, floating bodies and $K$-dense sets.

In order to prove Theorem 1.1 we closely follow Petty's strategy. Petty's argument was based on the observation that if $K$ is a smooth convex body satisfying (1.1) and we define $\bar{h}_{K}(x)=|x| h_{K}(x /|x|)$, then $\bar{h}_{K}^{2}$ is a solution of the Monge-Ampère equation

$$
\operatorname{det} \frac{1}{2} D^{2} \bar{h}_{K}^{2}(x)=c_{K}, \quad x \in \mathbf{R}^{N} .
$$

Combining this remark with classical results due to Pogorelov, $[14,15,16]$, Petty proved that $\bar{h}_{K}^{2}$ is a quadratic polynomial and hence that $K$ is an ellipsoid. By an approximation procedure we show that if a convex set $K$ satisfies (1.1), then its support function still satisfies (1.2) in the Aleksandrov sense, see Section 2.2 for the definition. By relying on standard techniques one can then show that any Aleksandrov solution of (1.2) is smooth and hence, by Pogorelov's Theorem, a quadratic polynomial.

The paper is organized as follows: in Section 2 we recall some preliminaries concerning Convex Geometry and weak solution of the Monge-Ampère equation, in Section 3 we provide a proof of Theorem 1.1.

After we finished writing this note, Prof. Schneider informed us that in the new edition of his book, there is a sketch of the proof of Theorem 1.1 based on Caffarelli's regularity results for the solutions of the Minkowski problem, see the Remark after Theorem 10.5.1 in [20].

\section{Prelimanaries}

In this section we recall some basic notions concerning convex bodies and solutions of the Monge-Ampère equation.

2.1. Convex Geometry. We denote by $\mathscr{K}^{N}$ the set of convex bodies (a convex body is a compact convex set with nonempty interior) of $\mathbf{R}^{N}$. We can associate a convex body $K$ with a measure $\mu_{K}$ supported on the unit sphere, 
called the surface area measure, with the property that, for every Borel set $A \subset \mathbf{S}^{N-1}, \mu_{K}(A)$ is the $(N-1)$-dimensional Hausdorff measure of the set of the points in the boundary of $K$ whose normal cone has nonempty intersection with $A$. More precisely, if for $x \in \partial K$, we define the possibly multivalued map

$$
N_{K}(x)=\left\{\omega \in \mathbf{S}^{N-1}: \omega \cdot(y-x) \leq 0 \text { for all } y \in K\right\},
$$

then

$$
\mu_{K}(A)=\mathscr{H}^{N-1}\left(N_{K}^{-1}(A)\right) .
$$

It is possible to show (see [19, Proposition 4.10]) that such measures are continuous in the $K$-variable with respect to the Hausdorff convergence. Namely

$$
\lim _{i} \int_{\mathbf{S}^{N-1}} \varphi d \mu_{K_{i}}=\int_{\mathbf{S}^{N-1}} \varphi d \mu_{K},
$$

for every $\varphi \in C\left(\mathbf{S}^{N-1}\right)$, whenever $K_{i} \rightarrow K$ in the Hausdorff distance.

When $K$ is $C_{+}^{2}$, i.e. if it is $C^{2}$-regular body with strictly positive GaussKronecker curvature $\kappa$, the surface area measure is absolutely continuous with respect to the Hausdorff measure $\mathscr{H}^{N-1}\left\llcorner\mathbf{S}^{N-1}\right.$ and its density is given by $\kappa \circ N_{k}^{-1}$ (note that $N_{K}$ is single valued and injective since $K$ has differentiable boundary and it is strictly convex).

A convex body $K$ is said to possess a curvature function provided there exists a positive and continuous function $f_{K}: \mathbf{S}^{N-1} \rightarrow \mathbf{R}$ such that ${ }^{2}$

$$
\mu_{K}=f_{K} \mathscr{H}^{N-1}\left\llcorner\mathbf{S}^{N-1} .\right.
$$

Conversely given a positive and continuous function $f: \mathbf{S}^{N-1} \rightarrow \mathbf{R}$, Minkowski existence and uniqueness Theorem, $[2,10,11,12,15]$, asserts that, provided $f$ fulfills the following (necessary) condition

$$
\int_{\mathbf{S}^{N-1}} \omega f(\omega) d \mathscr{H}^{N-1}(\omega)=0,
$$

there exists a unique (up to translation) convex body $K$ whose curvature function equals $f$.

The above condition leads us to the following observation: while the lefthand side of (1.1) is invariant under translations of $K$ the right-hand is affected by translations. However we shall note that, for every convex body $K$, there exists a point, say $p_{K}$, such that $h_{K-p_{K}}^{-N-1}$ is a curvature function. To see this let us recall that the polar reciprocal of a convex body $K$ with respect to the point $p$ is definend as

$$
K_{p}^{*}=\left\{x \in \mathbf{R}^{N}: x \cdot(y-p) \leq 1 \text { for all } y \in K\right\} .
$$

\footnotetext{
${ }^{2}$ In [13] mixed volumes are used to define curvature functions, however the definition given by Petty coincide with the one above by virtue of [20, Theorem 4.2.3].
} 
One can show that there exists a unique point, $p_{K}$, such that

$$
V\left(K_{p_{K}}^{*}\right)=\min \left\{V\left(K_{p}^{*}\right): p \in \mathbf{R}^{N}\right\},
$$

this point is the Santalo point of $K$. We denote by $K^{*}$ the set $K_{p_{K}}^{*}$. It is wellknown that the polar reciprocal of a convex body with respect to its Santalò point has its barycenter at the origin (see [17]), this implies that

$$
\int_{\mathbf{S}^{N-1}} \omega \rho_{K^{*}}^{N+1}(\omega) d \mathscr{H}^{N-1}(\omega)=0
$$

where $\rho_{K^{*}}$ denotes the radial function of the convex set $K^{*}$, i.e. $\rho_{K^{*}}(\omega)=$ $\sup \left\{\lambda: \lambda \omega \in K^{*}\right\}$. Since one can easily show that $h_{K}=1 / \rho_{K^{*}}$, then $h_{K}^{-N-1}$ satisfies condition (2.4) and hence, by Minkowski Theorem, for every $K \in \mathscr{K}^{N}$, there exists a body $K^{\prime}$ such that $f_{K^{\prime}}=h_{K-p_{K}}^{-N-1}$.

From these considerations we note that, if we define a map $\Lambda$, from the set of convex bodies whose Santalo point is the origin in itself, associating each convex body $K$ the solution of the Minkoski problem with data $h_{K}^{-N-1}$, then $K$ is a solution of (1.1) if and only if its image $\Lambda(K)$ is a dilation of $K$. We refer the reader to [6] for more details.

2.2. Aleksandrov solutions of the Monge-Ampère equation. In this section we recall the notion of Aleksandrov solutions of the Monge-Ampère equation and we summarize the properties of these solutions which we will need in the sequel, see $[3,5]$ for a more detailed exposition.

Let $u$ be a convex function defined on a convex open domain $\Omega \subset \mathbf{R}^{N}$, the subdifferential of $u, \partial u$, is the multi-valued map given by

$$
\partial u(x)=\left\{p \in \mathbf{R}^{N}: u(y) \geq u(x)+p y \cdot(y-x), \forall y \in \Omega\right\} .
$$

We define a measure $v_{u}$, and we call it Monge-Ampère measure of $u$, as follows: for a Borel set $A \subset \Omega$

$$
v_{u}(A)=V(\partial u(A)):=V\left(\bigcup_{x \in A} \partial u(x)\right) .
$$

Note that if $u \in C^{2}$, the change of variable formula gives that $d v_{u}=\operatorname{det} D^{2} u d x$. We then call $u$ an Alexandrov solution of the equation

$$
\operatorname{det} D^{2} u=f
$$

provided $v_{u}=f d x$. Among several properties of Aleksandrov solutions we are going to use the following concerning their stability under uniform limit, see [5, Lemma 1.2.3] for a proof.

LeMma 2.1. If $u_{k}$ are convex functions defined on an open set $\Omega$ and $u_{k} \rightarrow u$ uniformly, then

$$
v_{u_{k}} \stackrel{*}{\rightarrow} v_{u}
$$


as Radon measures in $\Omega$, that is

$$
\int \varphi d v_{u_{k}} \rightarrow \int \varphi d v_{u} \quad \forall \varphi \in C_{c}^{0}(\Omega)
$$

By relying on the uniqueness of the Aleksandrov solution to the Dirichlet problem for the Monge-Ampère equation, [5, Corollary 1.4.7] and on their stability under uniform limits, one can prove the following classical theorem. For the sake of completeness we sketch the main steps of its proof, see also [3, Section 2] for a more detailed account.

THEOREM 2.2. Let $u$ be a strictly convex function defined on a convex set $\Omega$ satisfying

$$
v_{u}=f d x \text { in } \Omega
$$

If $f \in C^{\infty}(\Omega)$ and $\lambda \leq f \leq \Lambda$ for some $\lambda, \Lambda>0$, then for every $\Omega^{\prime} \Subset \Omega$, $u \in C^{\infty}\left(\Omega^{\prime}\right)$.

Proof. Fix $x_{0} \in \Omega^{\prime}, p \in \partial u\left(x_{0}\right)$, and consider the section of $u$ at height $t$ defined as

$$
S(x, p, t):=\{y \in \Omega: u(y) \leq u(x)+p \cdot(y-x)+t\} .
$$

Since $u$ is strictly convex we can choose $t>0$ small enough so that $S\left(x_{0}, p, t\right) \Subset$ $\Omega^{\prime}$. Then we consider a sequence of smooth uniformly convex sets $S_{i}$, converging to $S\left(x_{0}, p, t\right)$ and we apply classical continuity methods in order find a function $v_{i} \in C^{\infty}\left(S_{\varepsilon}\right)$ solving

$$
\begin{cases}\operatorname{det} D^{2} v_{i}=f * \varrho_{\varepsilon_{i}} & \text { in } S_{i} \\ v_{i}=0 & \text { on } \partial S_{i}\end{cases}
$$

where $\varrho_{\epsilon_{i}}$ is a sequence of mollifying kernels, see [3, Theorem 2.11] and [4, Chapter 17]. We apply Pogorelov estimates, see for instance [3, Theorem 2.12], to $v_{i}$ to infer that

$$
\left|D^{2} v_{i}\right| \leq C \quad \text { in } S\left(x_{0}, p, t / 2\right) \Subset S\left(x_{0}, p, t\right)
$$

for a constant $C$ independent on $i \in \mathbf{N}$. Since $S_{i} \rightarrow S\left(x_{0}, p, t\right)$ and $u(x)=$ $u\left(x_{0}\right)+p \cdot x+t$ on $\partial S\left(x_{0}, p, t\right)$, by stability and uniqueness of weak solutions we deduce that $v_{i}+u\left(x_{0}\right)+p \cdot x+t \rightarrow u$ uniformly as $i \rightarrow \infty$, hence $\left|D^{2} u\right| \leq C$ in $S\left(x_{0}, p, t / 2\right)$. This makes the Monge-Ampère equation uniformly elliptic, hence Evans-Krylov Theorem and Schauder theory imply that $u \in C^{\infty}\left(S\left(x_{0}, p, t / 4\right)\right)$, see [4, Chapter 17]. By the arbitrariness of $x_{0}$ we obtain that $u \in C^{\infty}\left(\Omega^{\prime}\right)$, as desired.

By a well-known example, strict convexity of $u$ is necessary in order to prove the above Theorem. The following result, due to Caffarelli, implies that the 
obstruction to strict convexity can only arise from the boundary behavior. In particular every entire solution has to be strictly convex. We recall that $x$ is an extremal point of a convex set $K$ if $x \in \bar{K}$ and $K \backslash\{x\}$ is convex.

THEOREM 2.3 ([1]). Let $\Omega$ be an open convex and let $u$ be a convex function such that

$$
\lambda d x \leq v_{u} \leq \Lambda d x
$$

for some $\lambda, \Lambda>0$. For every $x \in \Omega$ and $p \in \partial u(x)$, if the set

$$
\Gamma_{x, p}:=\{y \in \Omega: u(y)=u(x)+p \cdot(y-x)\}
$$

contains more than one point, then it has no extremal points in $\Omega$.

An easy corollary of the above theorem is the following:

COROllary 2.4. Let $u: \mathbf{R}^{N} \rightarrow \mathbf{R}$ be a convex function such that

$$
\lambda d x \leq v_{u} \leq \Lambda d x
$$

for some $\lambda, \Lambda>0$, then $u$ is strictly convex.

Proof. Let us assume by contradiction that for some $x_{0} \in \mathbf{R}^{N}$ and $p_{0} \in \partial u\left(x_{0}\right)$ the set $\Gamma_{x_{0}, p_{0}}$ contains more than one point, then according to Caffarelli's Theorem it must contain a line. Up to subtracting a linear function and to change the coordinates we can then assume that $u \geq 0$ and $u=0$ on

$$
\ell:=\left\{x \in \mathbf{R}^{N}: x=\left(x_{1}, 0, \ldots, 0\right)\right\} .
$$

This easily implies that $\partial u\left(\mathbf{R}^{N}\right) \subset e_{1}^{\perp}$ and hence that $v_{u}=0$, contradicting (2.7).

\section{Proof of the main theorem}

In this section we prove Theorem 1.1, the argument is based on an approximation procedure in order to show that, for a convex body satisfying (1.1), $\bar{h}_{K}^{2} / 2$ is an Aleksandrov solution of (1.2). At this point we can apply Corollary 2.4 and Theorem 2.2 to show that $\bar{h}_{k}^{2}$ is smooth and hence the classical Pogorelov argument can be applied. More in general we prove the following:

THEOREM 3.1. Let $K$ be a convex body which possesses a curvature function $f_{K}$ and let $\bar{h}_{K}$ be the one-homogeneous extension of its support function, $\bar{h}_{K}=$ $|x| h_{K}(x /|x|)$, then

$$
\operatorname{det} \frac{1}{2} D^{2} \bar{h}_{K}^{2}=f_{K}\left(\frac{x}{|x|}\right) h_{K}^{N+1}\left(\frac{x}{|x|}\right) d x \text { in } \mathbf{R}^{N}
$$

in the Aleksandrov sense. 
In order to prove the above Theorem we need to approximate, in the Hausdorff topology, a convex body with $C_{+}^{2}$ bodies, for which we know that (3.8) holds true at least in $\mathbf{R}^{N} \backslash\{0\}$. We know from an old theorem by Minkowski that convex sets with analytic boundary are dense in $\mathscr{K}^{N}$, several years later Schmuckenschläger (see [18]) gave a simple proof of the theorem and showed that one can explicitly write down an approximating sequence with further additional properties; more precisely we have

TheOREM 3.2 ([18]). Let $K$ a convex body, there exist a sequence $\left\{K_{i}\right\}_{i \in \mathbf{N}}$, $K_{i+1} \subseteq K_{i}$, such that

- $K_{i}$ and $K_{i}^{*}$ have real analytic boundaries,

- The Gaussian curvature of both $K$ and $K^{*}$ is strictly positive,

- $K_{i} \rightarrow K$ in the Hausdorff distance.

We now discuss the proof of Theorem 3.1.

Proof of Theorem 3.1. We divide the proof in three steps:

- Step 1: Equation (3.8) holds true if $K \in C_{+}^{2}$. Let $K \in C_{+}^{2}$, then $h_{K} \in C^{2}$ and $\bar{h}_{K} \in C^{2}\left(\mathbf{R}^{N} \backslash\{0\}\right)$. Then a classical computation, see [13, Lemma 8.4], implies that

$$
\operatorname{det} \frac{1}{2} D^{2} \bar{h}_{K}^{2}(x)=f_{K}\left(\frac{x}{|x|}\right) h_{K}^{N+1}\left(\frac{x}{|x|}\right) \quad \forall x \in \mathbf{R}^{N} \backslash\{0\} .
$$

In particular, by the change of variable formula, if we denote by $v_{K}$ the MongeAmpére measure of $\bar{h}_{K}^{2}$

$$
v_{K}=f_{K}\left(\frac{x}{|x|}\right) h_{K}^{N+1}\left(\frac{x}{|x|}\right) d x
$$

as Radon measures on $\mathbf{R}^{N} \backslash\{0\}$. Moreover since $\bar{h}_{K}^{2}$ is homogeneous of degree two, it is differentiable in 0 and $\partial \bar{h}_{K}^{2}(0)=\{0\}$. Recalling the definition of MongeAmpère measure (2.5), we then see that for every Borel set $A \subset \mathbf{R}^{n}$

$$
\begin{aligned}
v_{K}(A) & =v_{K}(A \backslash\{0\})+v_{K}(\{0\}) \\
& =v_{K}(A \backslash\{0\})+V(\{0\})=v_{K}(A \backslash\{0\}) .
\end{aligned}
$$

Hence (3.2) is valid (as equality between measures) also in $\mathbf{R}^{N}$.

- Step 2: Let $K_{i}$ be a sequence of convex bodies for which Theorem 3.1 is valid and let $K$ be a convex body admitting a curvature function $f_{K}$. If $K_{i} \rightarrow K$ in the Hausdroff distance, then the conclusion of the Theorem 3.1 holds true for $K$.

Since $K_{i} \rightarrow K$ in the Hausdorff distance, $h_{K_{i}} \rightarrow h_{K}$ uniformly on $\mathbf{S}^{N-1}$ and $\bar{h}_{K_{i}}^{2} \rightarrow \bar{h}_{K}^{2}$ locally uniformly in $\mathbf{R}^{N}$. According to Lemma 2.1 it is enough to show that

$$
v_{K_{i}}=f_{K_{i}}\left(\frac{x}{|x|}\right) h_{K_{i}}^{N+1}\left(\frac{x}{|x|}\right) d x \stackrel{*}{\rightarrow} f_{K}\left(\frac{x}{|x|}\right) h_{K}^{N+1}\left(\frac{x}{|x|}\right) d x
$$


as Radon measures in $\mathbf{R}^{N}$. To this end let $\varphi \in C_{c}^{0}\left(\mathbf{R}^{N}\right)$ and note that for every $\varrho \in[0,+\infty), \quad \mathbf{S}^{N-1} \ni \omega \mapsto \varphi(\varrho \omega)$ is continuous. Since $h_{K_{i}} \rightarrow h_{K}$ uniformly on $\mathbf{S}^{n-1}$ and

$$
\int \varphi d v_{K_{i}}=\int_{0}^{\infty} \varrho^{N-1} \int_{\mathbf{S}^{N-1}} \varphi(\varrho \omega) f_{K_{i}}(\omega) h_{K_{i}}(\omega) d \mathscr{H}^{N-1}(\omega),
$$

an application of Lebesgue Dominated Convergence Theorem (recall that $\varphi$ is compactly supported) shows that in order to prove (3.9) it is enough to show that

$$
f_{K_{i}}(\omega) d \mathscr{H}^{N-1} \stackrel{*}{\rightarrow} f_{K}(\omega) d \mathscr{H}^{N-1}
$$

as Radon measures on $\mathbf{S}^{N-1}$. This however follows by the continuity of curvature measures under the Hausdorff convergence, (2.3).

- Step 3: Conclusion. If $K$ is a convex body admitting a curvature function we can apply Theorem 3.2 to approximate it with a sequence of convex bodies $K_{i} \in C_{+}^{2}$, by Step 1 the conclusion of the Theorem holds true for $K_{i}$ and hence by Step 2 also for $K$.

Proof of Theorem 1.1. According to Theorem 3.1, if $K$ is a convex body satisfying (1.1), then

$$
\operatorname{det} \frac{1}{2} D^{2} \bar{h}_{K}^{2}=c_{K} d x \quad \text { on } \mathbf{R}^{N}
$$

in the Aleksandrov sense. By Corollary $2.4, \bar{h}_{K}^{2}$ is strictly convex and by Theorem 2.2, $\bar{h}_{K}^{2} \in C^{\infty}\left(\mathbf{R}^{N}\right)$. By applying the classical Pogorelov argument (see [5, Theorem 4.3.1] for a proof) $\bar{h}_{K}^{2}(x)=A x \cdot x$ for some positive symmetric matrix $A$, which immediately implies that $K$ is an ellipsoid.

\section{REFERENCES}

[1] L. A. Caffarelli, A localization property of viscosity solutions to the Monge-Ampère equation and their strict convexity, Ann. of Math. (2) 131 (1990), 129-134.

[2] S. Y. Cheng and S. T. YAu, On the regularity of the solution of the $n$-dimensional Minkowski problem, Comm. Pure Appl. Math. 29 (1976), 495-516.

[3] G. De Philippis and A. Figalli, The Monge-Ampère equation and its link to optimal transportation, to appear in Bull. Amer. Math. Soc.

[4] D. Gilbarg and N. S. Trudinger, Elliptic partial differential equations of second order, Springer-Verlag, New York, 1983.

[ 5 ] C. Gutierrez, The Monge-Ampère equation, Progress in nonlinear differential equations and their applications 44, Birkhäuser Boston, Inc., Boston, MA, 2001.

[6] E. LutwaK, Selected affine isoperimetric inequalities, Handbook of convex geometry (P. M. Gruber and J. M. Wills, eds.), North-Holland, Amsterdam, 1993, 151-176.

[ 7 ] R. Magnanini AND M. Marini, Characterization of ellipsoids as K-dense sets, 2013, preprint.

[8] R. Magnanini and M. Marini, Characterization of ellipses as uniformly dense sets with respect to a family of convex bodies, to appear in Ann. Mat. Pura Appl.

[9] M. Meyer, S. Reisner AND M. Schmuckenschläger, The volume of the intersection of a convex body with its translates, Mathematika 40 (1993), 278-289. 
[10] H. Minkowski, Allgemeine lehrsätze über die konvexen polyeder, Nachr. Ges. Wiss. Göttingen, 1887, 103-121.

[11] H. Minkowski, Volumen und oberfläche, Math. Ann. 57 (1903), 447-495.

[12] L. Nirenberg, The Weyl and Minkowski problems in differential geometry in the large, Comm. Pure App. Math. 6 (1953), 337-394.

[13] C. M. Petty, Affine isoperimetric problems, Ann. N.Y. Acad. Sci. 440 (1985), 113-127.

[14] A. V. Pogorelov, On the improper convex affine hyperspheres, Geometriae Dedicata 1 (1972), 33-46.

[15] A. V. Pogorelov, The Minkowsky multidimensional problem, Scripta series in mathematics, V. H. Winston \& Sons, Washington, D.C., 1978.

[16] A. V. Pogorelov, Regularity of generalized solutions of the equation $\operatorname{det}\left(u_{i j}\right) \theta(\nabla u, u, x)=$ $\varphi(x)$, Dokl. Akad. Nauk SSSR 275 (1984), 26-28.

[17] A. Santalò, Un invariante afin para los cuerpos convexos del espacio de $n$ dimensiones, Port. Math. 8 (1949), 155-161.

[18] M. Schmuckenschläger, A simple proof of an approximation theorem of H. Minkowski, Geom. Dedicata 48 (1993), 319-324.

[19] R. Schneider, Curvature measures of convex bodies, Ann. Mat. Pura Appl. 116 (1978), 101-134.

[20] R. SchneIder, Convex bodies: the Brunn-Minkovski theory, Second Edition, Cambridge University Press, Cambridge, 2014.

[21] A. Stancu, The floating body problem, Bull. London Math. Soc. 38 (2006), 839-846.

\section{Michele Marini}

Scuola Normale Superiore

Piazza dei Cavalieri 7

56126 PISA

ITALY

E-mail: michele.marini@sns.it

Guido de Philippis

INSTITUT FÜR MATHEMATIK UNIVERSITÄT ZÜRICH

WINTERTHURERSTR. 190

CH-8057 ZÜRICH

SwITZERLAND

E-mail: guido.dephilippis@math.uzh.ch 